Стоянов Н.И., Северо-Кавказский федеральный университет Воронин А.И., г. Ставрополь, Россия,

Шапошников E.B. flasherel@rambler.ru

\title{
СОВЕРШЕНСТВОВАНИЕ ТЕХНОЛОГИИ ОБРАБОТКИ ВЫСОКО- МИНЕРАЛИЗОВАННЫХ ВОД НА ОСНОВЕ ТЕОРИИ ПЛАНИРОВАНИЯ ЭКСПЕРИМЕНОВ ПРИ ТЕПЛОВЫХ МЕТОДАХ ПОВЫШЕНИЯ НЕФТЕОТДАЧИ
}

Введение:

Актуальность исследования обусловлена проблемой дефицита запасов пресных вод в ряде регионов России и индустриальных центрах. Дефицит пресной воды во многих отраслях промышленности может быть восполнен за счет использования сточных вод, подвергнутых, предварительно, соответствующей обработке. В решении стратегических задач в сфере несттегазового комплекса России по увеличению объемов добычи углеводородов особое место занимает такой глобальный сырьевой ресурс, как вода. В связи с этим, статья направлена на совершенствование методов подготовки питательной воды для парогенераторов, используемых для паротеплового воздействия на нефтяные пласты с целью повышения их коэффициента нефтеотдачи.

Материалы и методы: В многообразии методов умягчения воды, как правило отдают предпочтение наиболее экономичным и безопасным с экологической точки зрения. Приводя в пример метод непрерывного многоступенчатого умягчения на основе технологии фильтров непрерывного действия (ФНД) Анализ методов и схем ионообменной обработки минерализованных вод показывает, что глубокое умягчение по методу $\mathrm{Na}$ - катионирования получило широкое развитие в силу следующих технологических преимуществ.

Результать и обсуждение:

Выводы:
Известны способы, когда требуется проведение нескольких повторных опытов для выбора основных наиболее значимых фракторов, оказывающих влияние на проведение эксперимента, отбрасывая ложные значение не поддающиеся систематизации, получают достоверный результат. Как правило для упрощения расчетных значений и числа изысканий выбирают метод априорной информации. Применение метода позволяет проводить ранжирование всех фракторов. Избыточные значения сводятся к минимуму, недостаточные к максимуму по контрольным показателям

Полученные экспериментальные данные по исследованию процесса регенерации с учетом влияния четырех определяющих факторов, а также выработанные в ходе их анализа рекомендации позволяют перейти к разработке новых эффективных схем установок ионообменной обработки воды. Материалы статьи представляют практическую ценность для нефтегазового комплекса России.

умягчение высокоминерализованных вод, теплоэнергетика, интенсификация нефтеотдачи, планирование эксперимента. 


\title{
Stoyanov N.I. Voronin A.I. Shaposhnikov E.V.
}

\section{Perfecting of technology of processing highly mineralized waters on the basis theories of scheduling eksperimenov at thermal methods of increase in oil recovery}

\begin{abstract}
The relevance of a research is caused by a problem of deficiency of reserves of sweet waters in a number of regions of Russia and the industrial centers. The shortage of sweet water in many industries can be filled due to use of the sewage subjected, beforehand, to the corresponding processing. In the solution of strategic tasks in the sphere of an oil and gas complex of Russia of increase in volumes of extraction of hydrocarbons a specific place is held by such global raw resource as water. In this regard, article is directed to perfecting of methods of preparation of feedwater for the steam generators used for steam impact on oil layers for the purpose of increase in their
\end{abstract}

Materials

and methods: In variety of methods of water demineralizing, as a rule give preference to the most economic and safe from the ecological point of view. Citing as an example a method of the continuous multistage softening on fundamentals of technology of the filters of the continuous action (FCA) the Analysis of methods and schemes of ion-exchange processing of salt waters shows that deep softening by the Na method - cation exchange received broad development owing to the following technological advantages.

Results and discussion: Ways when carrying out several repeated experiences for the choice of the major most significant factors affecting on carrying out an experiment is required are known, rejecting chance value not giving in systematizations, receive reliable result. As a rule, for simplification of calculated values and number of researches choose a method of the prior information. Application of a method allows to carry out ranging of all factors. Excess values are minimized, poor to a maximum on control indicators.

Conclusion: $\quad$ The obtained experimental data on a research of a protests of regeneration taking into account influence of four defining factors and also the recommendations developed during their analysis to pass to development of new effective schemes of installations of ion-exchange water treatment

$\begin{array}{ll}\text { Keywords: } & \text { Materials of article are of practical value for an oil and gas complex of Russia. } \\ \text { softening of highly mineralized waters, power system, oil recovery }\end{array}$ intensification, scheduling of an experiment.

\section{ВВЕДЕНИЕ}

В современных условиях индустриализации общества, роста объемов промышленного производства, развития энергетики и повышения уровня комфортности среды обитания человека, проблемы водоснабжения населения и индустриальных центров приобретают все нарастающую остроту.

В настоящее время дефицит пресной воды ощущается в ряде экономических районов России, среди которых в первую очередь следует отметить такие как Дальний Восток, районы Крайнего Севера, Северный Кавказ.

Обеспечение доступных водных ресурсов одна из основных задач по стабилизации экономического развития страны. Повышение уровня социаль- 
ного благополучия не может происходить без развития современных энергоемких и общедоступных технологий.

Получение горячей воды или пара из высокоминерализованных вод происходит в условиях пересыщения нагреваемой воды по накипеобразователям и растворенным газам. Нестабильная вода может давать отложения не только в зоне подогрева, но и в трубопроводах подачи теплоносителя и теплоиспользующем оборудовании.

Разработка эффективных схем теплоэнергетических установок для получения пара и обессоленной воды из высокоминерализованных природных и сточных вод стала жизненной необходимостью как для теплоэнергетики, так и для большинства промышленных предприятий.

Анализ состояния вопросов приготовления горячей воды и пара, а также уровня технических разработок в области новых способов обессоливания природных высокоминерализованных вод $[1,2]$ показывает, что наиболее целесообразным решением вопроса приготовления теплоносителей для закачки в нефтяные пласты является разработка новых схем получения горячей высокоминерализованной воды.

Рассматривая методы опреснения, осуществляемые дистилляцией, обратным осмосом, электродиализом, химическим вымораживанием и химическим обессоливанием, сущность которых сводится к полному удалению растворенных солей, а также методы умягчения, заключающиеся в удалении отдельных (накипеобразующих компонентов), следует отметить, что все они сопряжены с высокими энергетическими затратами и стоимостью энергетического оборудования

Обширный опыт, накопленный в области технологии ионного обмена, подтверждает принципиальную возможность глубокого умягчения высокоминерализованных вод методом натрий-катионирования и обеспечения безнакипного режима работы испарителей и парогенераторов среднего и высокого давления при питании их умягченной высокоминерализованной водой $[7,24-28,89,90]$. Последнее может быть реализовано в условиях многоступенчатого умягчения и регенерации фильтров большим количеством регенерационного раствора с малой исходной жесткостью (2-3 мг-экв/л) расход которого пропорционален увеличению жесткости исходной воды. В тоже время необходимо отметить, что с ростом солесодержания исходной воды использование метода в рамках существующих схем приводит к снижению его эффективности. Последнее объясняется увеличением затрат на реагенты и ростом объемов сбросных вод, утилизация которых проблематична.

Однако, установки ионообменного умягчения выгодно отличаются от теплоиспользующего опреснительного оборудования, так как имеют ряд несомненных преимуществ. Для ионитных установок характерными отличиями являются: меньшая металлоемкость; высокая маневренность, позволяющая быстро менять производительность; меньшие капитальные затраты; возмож- 
ность работы при пониженных параметрах. Следовательно, для реализации преимуществ метода необходимо дальнейшее совершенствование существующих схем и разработка новых эффективных технологий.

Обзор экспериментальных и теоретических работ в области водоподготовки подтверждает принципиальную возможность непосредственного питания различных типов теплогенераторов глубоко умягчённой высокоминерализованной водой $[3,4,5]$.

При этом, в качестве основных вариантов их использования можно выделить следующие направления: приготовление питательной воды для водогрейных и паровых котлов, используемых при паротепловой обработке нефтяных пластов с целью повышения их коэффициента нефтеотдачи, водоподготовка для паровых и водогрейных котлов производственных и муниципальных котельных, а также технологические процессы химических и металлургических предприятий.

Теплогенераторы и все их конструктивные элементы, контактирующие с жидкой или газообразной средой теплоносителя, которая в рассматривасмом случае является агрессивной, за счет высокого содержания солей исходной воды. В том числе возникновение солевых отложений на поверхностях нагрева.

В настоящее время разработан ряд способов обработки минерализованных вод [6], включающих глубокое умягчение воды путем ее катионообменной обработки и регенерацией истощенного катионита продувочной водой парогенераторов.

Существенным преимуществом является:

a) уменьшение расхода реагентов

б) снижение стоимости и себестоимости электроэнергии.

в) увеличение срока службы оборудования.

г) снижение затрат при плановом и капитальном ремонте

Устройства для получения пара используют пресную низкоминерализованную воду, показатель жесткости которой не превышает 10 мг на 1 литр эквивалента, соответственно для использования утилизируемой води необходимо произвести ее деминерализацию до значений, допускающих использование этой воды.

\section{МАТЕРИАЛЫ И МЕТОДЫ}

В многообразии методов умягчения воды, как правило отдают предпочтение наиболее экономичным и безопасным с экологической точки зрения. Приводя в пример метод непрерывного многоступенчатого умягчения на основе технологии фильтров непрерывного действия (ФНД) с неподвижным слоем ионита достигаются удовлетворительны результаты [7]. Они обеспечивают более полное использование регенерационного раствора за счет применения ступенчато-противоточной схемы регенерации, а также 
полное «срабатывание» располагаемой рабочей емкости поглощения ионита в цепи нескольких последовательно включенных фильтров, работающих в режиме полного истощения. В стандартных схемах водоподготовки фильтры периодического действия работают до так называемого «проскока жесткости», используя лишь часть располагаемой емкости катионита

Анализ методов и схем ионообменной обработки минерализованных вод показывает, что глубокое умягчение по методу $\mathrm{Na}$ - катионирования получило широкое развитие в силу следующих технологических преимуществ: использование для регенерации фильтров наиболее дешевого реагента (раствора $\mathrm{NaCl}$ ) и широко распространенных ионообменных материалов (преимущественно КУ-2 и сульфоугля);

удаление из исходного раствора лишь части ионов $\left(\mathrm{Ca}^{2+}\right.$ и $\mathrm{Mg}^{2+}$ ), являющихся накипеобразующими ионами жесткости; возможность использования для регенерации фильтров продувочного раствора испарителей, различных выпарных установок, парогенераторов, работающих на умягченной минерализованной воде.

Опыт практического применения методов натрий-катионирования в установках ионообменной обработки минерализованных вод показывает, что технологические показатели, а, следовательно, и технико-экономические характеристики установок этого типа находятся в сложной зависимости от составов исходной воды и режимных факторов регенерации.

Решающее значение среди них принадлежит таким как: удельный расход реагента; концентрация регенерационного раствора; скорость пропускания регенерационного раствора; состав истощенного катионита перед регенерацией, определяемый составом исходной воды. Основываясь на литературных данных, эта зависимость может быть выявлена лишь для отдельных факторов в определенном диапазоне их изменения, что затрудняет выбор схемы и расчет оптимального режима установок для различных составов исходной питательной воды.

Между тем, состав исходной воды, оказывающий, как отмечалось выше, наиболее сильное влияние на технологические показатели процесса, может меняться в очень широких пределах. Следовательно, совершенствование метода натрий - катионирования высокоминерализованных вод должно идти, очевидно, по пути обобщенно имеющихся данных на базе дальнейших экспериментальных и теоретических исследований.

Ввиду недостаточной разработанности теории динамики ионного обмена при высоких концентрациях рядом авторов предложены расчетные зависимости основных показателей процесса, полученные на основе обобщения результатов экспериментальных лабораторных и промышленных иссле- 
дований. Эти работы подтверждают возможность глубокого умягчения высокоминерализованных вод, а предлагаемые методы расчета позволяют определять основные технологические показатели работы установок для исследованного состава вод.

Однако, отмечая ценность накопленного опыта, следует отметить, что особенности сорбционной технологии при высоких концентрациях не позволяют распространить полученные данные за рамки проведенных исследований, а имеющиеся литературные данные не охватывают всего разнообразия составов высокоминерализованных природных и сточных вод. Из этого следует вывод о необходимости обобщения имеющихся литературных и новых экспериментальных данных на базе дальнейших теоретических исследований.

Техническому решению поставленной задачи препятствует сложность организации и проведения многофакторных экспериментальных лабораторных исследований по технологии умягчения высокоминерализованных вод в широком спектре их начальных концентраций. К тому же, их проведение требует значительных материальных затрат, связанных с закупкой высокотехнологичного оборудования и химических реагентов, а также времени на их проведение.

Каждый дополнительный фактор, включённый в программу исследований, приводит к значительному увеличению объёма экспериментальной работы. Например, при проведении полнофакторного эксперимента типа 2n увеличение числа факторов на единицу ведёт за собой удвоение числа опытов:

- при $\mathbf{n}=5 ; \mathrm{N}=2^{5}=32$ опыта;
- $\quad$ при $\mathbf{n}=6 ; \mathrm{N}=2^{6}=64$ опыта.

Очевидно, что для сокращения объёма экспериментальной части желательно иметь как можно меньшее число исследуемых факторов.

Однако, надо помнить и о том, что эксперименты могут потерять всякий смысл при исключении из программы исследований хотя бы одного фактора, оказывающего существенное влияние на целевую функцию.

То есть, сокращать программу экспериментов можно только за счёт исключения несущественных факторов. Для исключения, или отсеивания, факторов, не оказывающих существенного влияния на целевую функцию, необходимо заранее знать, какие факторы процесса и их взаимодействия являются наиболее значительными.

На первых этапах изучения процесса при отсутствии данных о значимости отдельных факторов рекомендуется включать в программу предварительных исследований все факторы, которые могут оказать влияние на функцию оптимизации. Теория планирования эксперимента располагает способами, позволяющими выделять существенные факторы из общего числа факторов системы [8] 


\section{РЕЗУЛЬТАТЫ ИССЛЕДОВАНИЙ И ИХ ОБСУЖДЕНИЕ}

Известны способы, когда требуется проведение нескольких повторных опытов для выбора основных наиболее значимых факторов, оказывающих влияние на проведение эксперимента, отбрасывая ложные значение не поддающиеся систематизации, получают достоверный результат. Как правило для упрощения расчетных значений и числа изысканий выбирают метод априорной информации.

Применение метода позволяет проводить ранжирование всех факторов. Избыточные значения сводятся к минимуму, недостаточные к максимуму по контрольным показателям.

\section{1. На основании литературных составляется перечень факто- ров, оказывающих влияние на исследуемый процесс, и ус- танавливаются области изменения каждого из них. \\ 2. Специалистам, работающим в данной области, предлагают расположить факторы в ряд по степени влияния их на це- левую функцию. При этом каждый специалист может до- полнить список, если он, по его мнению, являлся не пол- ным. При подборе специалистов руководствуются идеоло- гией наличия среди них представителей возможно больше- го числа научных школ. По результатам опроса составляет- ся матрица рангов. Обработку результатов опроса произво- дят по общепринятым методикам [9].}

Для каждого фактора определяется сумма рангов:

$$
\sum_{i=1}^{N} a_{j i}
$$

число опрошенных специалистов;

$a_{j i}-\quad$ ранг $j$-го фактора, присвоенный $i$-м исследователем.

Вычисляется отклонение $\Delta$ суммы рангов от средней суммы рангов для каждого из факторов:

$$
\Delta_{j}=\sum_{i=1}^{N} a_{j i}-\frac{1}{n} \sum_{j=1}^{n} \sum_{i=1}^{N} a_{j i}
$$

отклонение суммы рангов $\mathrm{j}$-го фактора от средней суммы рангов;

$\begin{array}{ll}\mathrm{n}- & \text { число факторов; } \\ \frac{1}{n} \sum_{j=1}^{n} \sum_{i=1}^{N} a_{j i}- & \text { средняя сумма рангов. }\end{array}$ 
Определив значения $\Delta_{j}$ для каждого из факторов, производится оценка согласованности мнений опрошенных специалистов. Для этого вводится коэффициент координации мнений $(\mathrm{W})$, который определяется по формуле:

$$
\begin{aligned}
W & =\frac{12 \times S}{N^{2}\left(n^{3}-n\right)} \\
\text { где } \quad S & =\sum_{j=1}^{n} \Delta_{j}^{2}
\end{aligned}
$$

По результатам выполненных вычислений в качестве определяющих факторов принимают те из них, которые определяют общую технологичность и экономичность установки водоподготовки. Это, как правило, состав обрабатываемой воды, удельный расход реагентов, скорости регенерации ионита и умягчения обрабатываемой воды.

Разработанные в настоящее время математические модели планов 1-го, 2-го и высших порядков позволяют описать с высокой точностью исследуемый процесс в широком интервале изменения определяющих факторов при минимальном количестве постановочных экспериментов.

Полученная математическая модель исследуемого процесса в виде уравнения регрессии 2-го или 3-го порядка может быть несколько упрощена за счет обнуления коэффициентов уравнения, признанных статистически незначимыми по критерию Стьюдента. Для практического использования полученного уравнения его следует проверить на адекватность исследуемому процессу по критерию Фишера и привести к инженерному виду, удобному для выполнения практических расчетов по определению технологических показателей установок умягчения высокоминерализованных вод.

По результатам анализа отдельные менее значимые члены уравнена были приняты разными нулю, а степень влияния других, более весомых, была усилена введением дополнительных членов уравнения. В итоге этих преобразований расчетное уравнение принимает вид:

$$
\begin{aligned}
& n_{p}=-1.5447-1.2304 d_{p}+0.006082 d_{p}^{2}+0.7397 \cdot \frac{d_{p}^{2}}{d_{p}+2}+2.4715 \sqrt{d_{p}}-0.01094 \cdot W_{p}-0.002894 \\
& \frac{1}{W_{p}-0.4}+0.1170 \frac{1}{d_{p} W_{p}}+0.7130 \cdot \alpha_{k o}-0.2793 \alpha_{k o}^{2}-0.08614 d_{p} \cdot \alpha_{k o}+ \\
& +0.004762 d_{p}^{2} \cdot \alpha_{k o}+0.1815 C_{p p}-0.04278 \cdot C_{p p}^{2}
\end{aligned}
$$

Средняя погрешность описания целевой функции по четырем факторам составляет $1,10 \%$.

Применив разработанную математическую модель на практике, авторами была составлена матрица центрального композиционного плана про- 
ведения экспериментов. Рассмотрев фильтры с неподвижным слоем ионита, можно проанализировать, получаемые на выходе значения путем исследования зависимости и разности параметров до и после фильтра. Математическое моделирование процессов позволяет создавать полноценную модель и отслеживать все стадии очистки в фильтрующем слое. Решение уравнений, описывающих статику и динамику ионного обмена, может быть выполнено в относительных коэффициентах, результатом которых является:

Результатом, описывающим статику ионного обмена и динамику процесса, является:

расчет равновесного состава катионита по заданному равновесному составу раствора;

расчет равновесного состава раствора по заданному равновесному составу катионита;

расчет рабочей обменной емкости поглощения катионита КУ-2-8 при натрий-катионитовом умягчении минерализованных вод;

расчет высоты рабочего слоя ионита в рабочей колонне при умягчении минерализованных вод на катионите КУ-2-8.

Последнее имеет принципиальное значение для оптимизации габаритных размеров ионообменных фильтров в установке непрерывного умягчения. А габариты фильтра непрерывного действия (ФНД) напрямую определяют ее экономическую целесообразность и сроки окупаемости.

В стандартных ионообменных фильтрах периодического действия высота слоя катионита определяется технологическими условиями меж регенерационного периода работы, который составляет от 8 до 12 часов. При этом, фронт переменной концентрации в отрегенерированном слое катионита непрерывно перемещается от верхней отметки слоя до нижней дренажной системы фильтра.

В фильтре непрерывного действия каждая ионообменная колонна находится в работе до полного истошения катионита, а проскок жесткости снимается в последующей ступени умягчения. А так как в режиме умягчения находятся одновременно 2 или 3 последовательно включенные колонны, то качество умягчения гарантированно высокое, а требуемый объем рабочей массы катионита сокращается 2-2,5 раза по сравнению с установками периодического действия.

Время работы катионита в каждой ионообменной колонне в режиме умягчения или регенерации сокращается до 1-2-х часов, что реально можно обеспечить только в автоматизированном режиме. На кафедре теплогазоснабжения и ЭН разработано гидравлическое устройство (многоходовой многопозиционный кран), обеспечивающее работу ФНД в непрерывном режиме без участия человека [9]. 
В современных условиях рыночной экономики реализация разработанной технологии и оборудования умягчения высокоминерализованных вод имеет реальные перспективы широкого внедрения не только северных нефтедобывающих районах страны, но и в ряде других регионов (прикаспийские районы и Крым), испытывающие дефищит в пресной воде.

\section{выводы}

Результаты проведенных экспериментальных исследований эффективности процесса регенерации по четырем определяющим факторам и их анализ позволяют выработать практические рекомендации по совершенствованию процесса регенерации катионита КУ-2-8 при глубоком умягчении высокоминерализованных вод и, в частности, воды Черного моря.

Полученные экспериментальные данные по исследованию процесса регенерации с учетом влияния четырех определяющих факторов, а также выработанные в ходе их анализа рекомендации позволяют перейти к разработке новых эффективных схем установок ионообменной обработки воды.

По результатам выполненных исследований получено уравнение, описывающее целевую функцию в широком интервале изменения 4-х определяющих факторов: удельный расход соли на регенерацию; скорость ввода регенерационного раствора; солесодержание и жесткость умягчаемой воды; концентрация регенерационного раствора.

Полученные научные результаты позволяют выполнять технологические расчеты режимов умягчения высокоминерализованных вод и, в частности, воды Черного моря, а также выполнять конструкторские разработки блоков химводоочистки ТЭЦ и производственно-отопительных котельных.

Выполненные предварительные технико-экономические расчеты подтверждают экономическую эффективность планируемых научно-исследовательских и конструкторско-технологических работ в рамках поставленной исследовательской задачи.

\section{БИБЛИОГРАФИЧЕСКИЙ СПИСОК}

1. Шищенко В.В., Седлов А.С., Федосеев Б.С. и др. Выбор оптимального метода водоподготовки для тепловых электростанций // Теплоэнергетика. 2005, №4. С. 54-60.

2. Шищенко В.В., Седлов А.С., Ильина И.П. и др. Опыт создания малоотходных систем водопользования на ТЭС // Теплоэнергетика. 2005, №4. С. 35-38.

3. Сень Л.И., Якубовский Ю. В. Парогенераторные установки на морской воде. Л.: Судостроение, 1979. 232 с.

4. Стоянов Н. И., Гейвандов И. А., Воронин А. И. и др. Совершенствование технологии получения питательной воды из 
высокоминерализованных вод для несртепромысловых парогенераторов // Нефтепромысловое дело. Ежемесячный научнотехнический журнал. М.: ВНИИОЭНГ, 2005, №11. С. 61-63.

5. Стоянов Н.И., Дзюбина М.А. Разработка технологии утилизации сточных вод для объектов нефтегазового комплекса // Вестник Северо-Кавказского федерального университета. г. Ставрополь: СКФУ, 2013, №2 (35) 282 с. (66-69).

6. Фейзиев Г. К. Высокоэффективные методы умягчения, опреснения и обессоливания воды. М.: Энергоатомиздат, 1988. 192 с.

7. Симонов П.П., Стоянов Н.И., Воронин А.И. Вопросы регулирования режима работы ФНД с неподвижным слоем ионита // Материалы XVIII конференции по итогам научно-исследовательской работы профессорско-преподавательского состава за 1988 г. Ставрополь: Изд. КМУ, СтПИ, 1989. С. 82-83.

8. Закгейм А. Ю. Введение в моделирование химико-технологических процессов. Математическое описание процессов. М.: Химия, 1973. 224 с.

9. Четыркин Е.М., Калихман И.Л. Вероятность и статистика. М.: Финансы и статистика, 1982. 319 с

10. Воронин А.И. и др. Многоходовой многопозиционный кран преимущественно для установок ионообменной очистки воды (авторское свидетельство). А.С. № 1691314 Государственный комитет СССР по делам изобретений и открытий, заявка № 4670249 от 30.03.90; Опубл. Бюл. № 42; 15.11.91. 6 с.

\section{REFERENCES}

1. Shishchenko V. V., Sedlov A. S., Fedoseyev B. S., etc. The choice of an optimum method of water treatment for thermal power plants, Thermal Engineering. - 2005, No. 4. Page 54-60.

2. Shishchenko V. V., Sedlov A. S., Ilyina I. P., etc. Experience of creation of low-waste systems of water use on thermal power plant, Thermal Engineering .2005, No. 4. Page 35-38.

3. Shade L. I., Yakubovsky Yu. V. Steam generating installations on ocean water. L.: Shipbuilding, 1979. 232 pages.

4. Stoyanov N.I., Geyvandov I. A., Voronin A. I., etc. Perfecting of technology of receiving feedwater from highly mineralized waters for oil-field steam generators, Oilfield Engineering . Monthly scientific and technical magazine. M.: JSC VNIIOENG, 2005, No.11. Page 61-63.

5. Stoyanov N.I., Dzyubina M.A. Development of technology of utilization of sewage for objects of an oil and gas complex, Messenger of the North Caucasian federal university. Stavropol: SKFU, 2013, No of 2 (35) 282 pages (66-69).

6. Feyziyev G.K. Highly effective methods of softening, desalting and desalting of water. M.: Energoatomizdat, 1988. 192 pages

7. Simonov P. P., Stoyanov N.I., Voronin A.I. Questions of regulation of 
a duty of FND with the fixed bed of an ionite // Materials of the XVIII conference following the results of research of the faculty for 1988 Stavropol. Prod. KMU, STPI, 1989. Page 82-83.

8. Zakgeym A. Yu. Introduction to model operation of chemical and technological processes. Mathematical description of processes. M.: Chemistry, 1973. 224 pages.

9. Chetyrkin E.M., Kalikhman I.L. Veroyatnost and statistics. M.: Finance and statistics, 1982. 319 pages.

10. Voronin A.I., etc. The multipass multiposition crane mainly for installations of ion-exchange water treatment (copyright certificate) Ampere-second. No. 1691314 the State committee of the USSR for inventions and opening, application No. 4670249 of 30.03 .90 Opubl. Bulletin No. 42; 15.11.91. 6 pages.

\section{Рукопись поступила в редакцию 17.01.19, принята к публикации 01.02.19}

\section{Сведения о6 авторах}

Стоянов Николай Иванович, д.Т.Н., профессор, заведующий кафедрой теплогазоснабжения и экспертизы недвижимости, инженерный институт, контакты (cnjzyjd1949@mail.ru, +79054113415).

Воронин Александр Ильич, к.т.н., доцент, доцент кафедры теплогазоснабжения и экспертизы недвижимости, инженерный институт контакты (cnjzyjd1949@mail.ru, +79054113415).

Шапошников Евгений Владимирович, ассистент кафедры теплогазоснабжения и экспертизы недвижимости, инженерный институт контакты (flasherel@rambler.ru, +79614459665).

\section{About the authors}

Stoyanov Nikolay Ivanovich, Dr.Sci.Tech., professor, head of the department of heatgas supply and examination of the real estate, engineering institute, contacts (cnjzyjd1949@mail.ru, 79054113415).

Voronin Alexander llyich, PhD in Technological Sciences, associate professor, associate professor of heatgas supply and examination of the real estate, engineering institute contacts (cnjzyjd1949@mail.ru 79054113415).

Shaposhnikov Evgeny Vladimirovich, assistant to department of heatgas supply and examination of the real estate, engineering institute contacts (flasherel@rambler.ru, 79614459665). 